\title{
Interaction between body mass index and serum uric acid in relation to blood pressure in community-dwelling Japanese men
}

\author{
Ryuichi Kawamoto ${ }^{1,2^{*}}$ (D), Daisuke Ninomiya ${ }^{1}$, Kensuke Senzaki ${ }^{1}$ and Teru Kumagi ${ }^{1}$
}

\begin{abstract}
Background: Few data is available on the association between body mass index (BMI), serum uric acid (SUA) levels and blood pressure (BP) categories in the disease continuum, when efforts for its prevention may be applicable.

Methods: We performed a cross-sectional study to examine the association between BMI, SUA and BP in a community-dwelling sample of Japanese men. Individuals not on antihypertensive and uric acid lowering medications, and aged 50 to 90 years [817men aged $66 \pm 9$ (mean \pm standard deviation) years] were recruited for the survey during a community based annual medical check-up. The main outcome was the presence of prehypertension [systolic BP (SBP) 120-139 mmHg and/or diastolic BP (DBP) 80-89 $\mathrm{mmHg}$ ] and hypertension [SBP $\geq 140$ and /or DBP $\geq 90$ ].

Results: In participants with a BMl of $<21.0 \mathrm{~kg} / \mathrm{m}^{2}$, increased SUA levels were positively associated with SBP and DBP, but in those with a BMI of $\geq 21.0 \mathrm{~kg} / \mathrm{m}^{2}$, increased SUA levels were negatively associated with SBP and DBP. The interaction between BMI and SUA as well as BMI and SUA was a significant and independent determinant for both SBP $(\beta=-1.125, p=0.001)$ and $\mathrm{DBP}(\beta=-0.995, p=0.005)$. Among participants, the respective prevalence of normotension, prehypertension, and hypertension was $19.5 \%$ and $53.7 \%$, and $19.8 \%$. The prevalence of normotension and prehypertension decreased with increasing BMI and the prevalence of hypertension increased with increasing BMI. In participants with a BMI $\geq 21.0 \mathrm{~kg} / \mathrm{m}^{2}$, the adjusted-odds ratio of SUA for hypertension was $0.75(95 \% \mathrm{Cl}, 0.59-0.95)$ compared with normotension and 0.82 (0.70-0.96) compared with prehypertension. In those with a BMl of $<21.0 \mathrm{~kg} /$ $\mathrm{m}^{2}$, these associations were not shown.
\end{abstract}

Conclusion: BMI may modify the association between SUA and blood pressure status among community-dwelling men.

Keywords: Blood pressure, Uric acid, Body mass index, Interaction, Risk factor, Community-dwelling men

\section{Background}

Hypertension is likely the most common disease in Japan and is strongly associated with an increased risk of cardiovascular disease (CVD). The Seventh Report of the Joint National Committee on Prevention, Detection, Evaluation and Treatment of High Blood Pressure (JNC 7) defined a systolic blood pressure (SBP) of 120 to $139 \mathrm{mmHg}$ and/or diastolic blood pressure (DBP) 80 to

\footnotetext{
* Correspondence: rykawamo@m.ehime-u.ac.jp

'Department of Community Medicine, Ehime University Graduate School of Medicine, Toon-city, Ehime 791-0295, Japan

${ }^{2}$ Department of Internal Medicine, Seiyo Municipal Nomura Hospital, 9-53 Nomura, Nomura-cho, Seiyo-city, Ehime 797-1212, Japan
}

$89 \mathrm{mmHg}$ as prehypertension [1] based on the evidence of a modestly increasing risk of CVD among individuals with such levels [2]. Many studies demonstrated that the prehypertensive group had a higher body mass index (BMI), central obesity, a family history of hypertension, a sedentary lifestyle, eating high sodium foods, smoking, excessive alcohol intake, impaired glucose tolerance/diabetes, higher levels of blood glucose, low-density lipoprotein cholesterol (LDL-C), and triglycerides (TG), and lower levels of high-density lipoprotein cholesterol (HDL-C) than the normotensive group [3-6]. The Jichi Medical School Cohort Study which enrolled 4706 males and 7342 females from among the general Japanese 
population suggested that $\mathrm{BMI}>23.0 \mathrm{~kg} / \mathrm{m}^{2}$ was the strongest determinant of prehypertension [7]. Thus lifestyle modification or even medical treatment is recommended for individuals with prehypertension [8].

Serum uric acid (SUA) is the end product of endogenous and dietary purine metabolism in humans, and is catalyzed by the enzyme xanthine oxidase, which is involved in producing reactive-oxygen species (ROS). Its excess accumulation can lead to various diseases [9]. SUA is closely associated with an increased risk of prehypertension [10, 11], hypertension [12], metabolic syndrome [13], and cardiovascular disease (CVD) [14, 15]. However, despite an association between serum SUA level and these conditions, SUA may not be considered a risk in these conditions [16], but rather as biologically inert or possibly anti-inflammatory because it can function as an antioxidant [17]. Increased SUA was significantly elevated in a linear manner. Moreover, increased BMI and weight loss may represent an effective nonmedical strategy for reducing SUA levels, especially in postmenopausal women and men [18]. Thus, the relationships between SUA and BP of participants categorized by BMI level may be different, and an interactive effect between BMI and SUA on blood pressure may be considered.

The aim of this study was to evaluate the prevalence of prehypertension and hypertension, and its association with BMI, SUA and other confounding factors \{e.g., age, habits, lipid, fasting plasma glucose (FPG), renal function, and liver function $\}$ using cross-sectional data from community-dwelling Japanese men aged of $\geq 50$ years.

\section{Methods}

\section{Study population}

The present study was designed as a part of the Nomura study [19]. Participants were selected through a community-based annual check-up process in a rural town located in Ehime prefecture, Japan. Baseline clinical characteristics including anthropometric parameters were obtained from the subject's personal health records of the evaluated medical check-up. Other characteristics such as smoking and alcohol habit, medication, and history of CVD were investigated by individual interviews using a structured questionnaire. After excluding individuals with antihypertensive and UA lowering medications, the final study sample included only eligible persons (Fig. 1). This study was approved by the ethics committee of Ehime University School of Medicine, and written informed consent was obtained from each subject.

\section{Evaluation of risk factors}

Information on demographic characteristics and risk factors was collected using clinical files. BMI was calculated

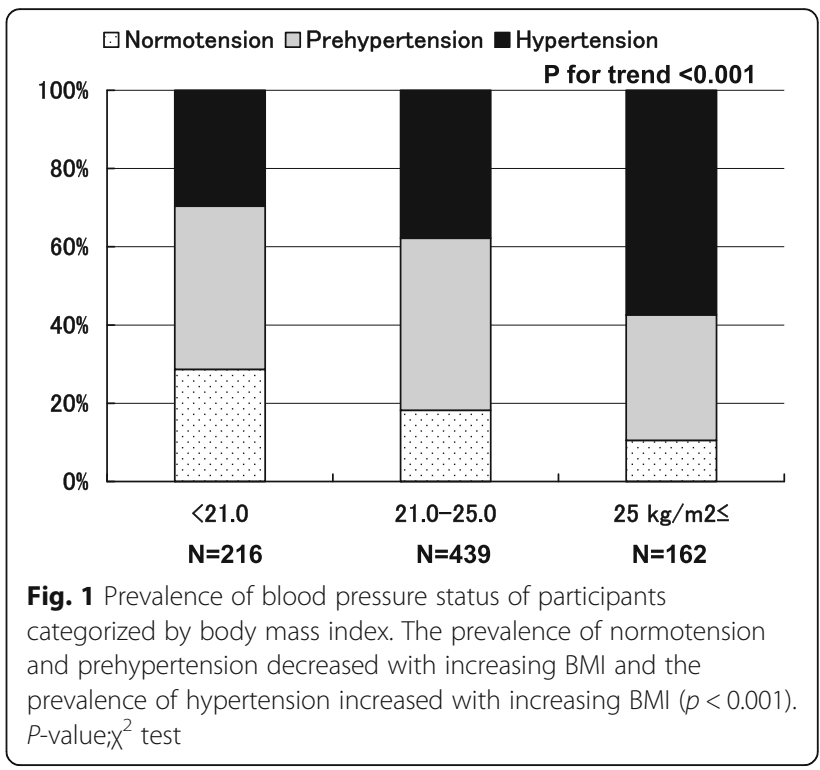

by dividing weight (in kilograms) by the square of height (in meters). Smoking status was defined as the number of cigarette packs per day multiplied by the number of years smoked (pack year), and the participants were classified into never smokers, past smokers, light smokers ( $<30$ pack year) and heavy smokers $(\geq 30$ pack year). Daily alcohol consumption was measured using the Japanese liquor unit in which a unit corresponds to $22.9 \mathrm{~g}$ of ethanol, and the participants were classified into never drinkers, occasional drinkers $(<1$ unit/day), light drinkers $(<2$ unit/ day), and heavy drinkers ( $\geq 2$ unit/day). We measured SBP and DBP in the right upper arm of participants in the sedentary position using an automatic oscillometric blood pressure recorder while the participants were seated after having rested for at least $5 \mathrm{~min}$. Appropriate cuff bladder size was determined at each visit based on arm circumference. Normotension was defined as not being on antihypertensive medication and having a SBP $<120 \mathrm{mmHg}$ and $\mathrm{DBP}<80 \mathrm{mmHg}$. Prehypertension was defined as not being on antihypertensive medication and having a SBP of 120 to $139 \mathrm{mmHg}$ and/or DBP of 80 to $89 \mathrm{mmHg}$. Hypertension was defined as SBP $\geq 140 \mathrm{mmHg}$ and/or DBP $\geq 90 \mathrm{mmHg}$ (9). Total cholesterol (T-C), TG, HDL-C, FPG, creatinine (enzymatic method), SUA, aspartate transaminase (ALT), and $\gamma$-glutamyl transpeptidase (GGT) were measured during fasting. LDL-C levels were calculated by the Friedewald formula (14). Participants with TG levels $\geq 400 \mathrm{mg} / \mathrm{dL}$ were excluded. The presence of diabetes and CVD was defined as a history of treatment for diabetes and CVD. Estimated glomerular filtration ratio (eGFR) was calculated using CKD-EPI equations modified by a Japanese 
coefficient: Male, $\mathrm{Cr} \leq 0.9 \mathrm{mg} / \mathrm{dl}, 141 \times(\mathrm{Cr} / 0.9)$ $0.411 \times 0.993$ age $\times 0.813 ; \mathrm{Cr}>0.9 \mathrm{mg} / \mathrm{dl}, 141 \times(\mathrm{Cr} / 0.9)$ $-1.209 \times 0.993$ age $\times 0.813$ [20].

\section{Statistical analysis}

Statistical analysis was performed using IBM SPSS Statistics Version 20 (Statistical Package for Social Science Japan, Inc., Tokyo, Japan). All values are expressed as mean \pm standard deviation (SD), unless otherwise specified. Data for TG, FPG, ALT, and GGT were skewed, and are presented as median (interquartile range) values, and were log-transformed for analysis. Differences in means and prevalence among the groups were analyzed by Student's t-test for continuous data and $x^{2}$ test for categorical data. Correlations between various characteristics and BP were determined using age-adjusted partial Pearson's correlation test and multiple linear regression analysis. Logistic regression analyses were used to test significant factors of prehypertension and hypertension (versus normotension), with prehypertension and hypertension serving as the dichotomous outcome variables, and age, BMI, alcohol consumption, smoking status, history of CVD, lipids, antilipidemic medication, FPG, antidiabetic medication, SUA, eGFR, ALT, and GGT as the confounding factors. The synergistic effect of BMI and SUA levels on blood pressure was evaluated using a general linear model. A value of $p<0.05$ was considered significant.

\section{Results}

The participants comprised 817 men aged $66 \pm 9$ years (range, 50-90) who do not take antihypertensive and UA lowering medications. Mean BMI in the study sample was $22.9 \mathrm{~kg} / \mathrm{m}^{2}$ (SD, 2.8), with $26.4 \%$ being underweight $\left(\mathrm{BMI}<21.0 \mathrm{~kg} / \mathrm{m}^{2}\right), 53.7 \%$ normal weight (BMI, 21.0 to $24.9 \mathrm{~kg} / \mathrm{m}^{2}$ ), $19.8 \%$ overweight or obese (BMI $\geq 25 \mathrm{~kg}$ / $\mathrm{m}^{2}$ ). Table 1 shows the background characteristics of participants categorized by BMI. BMI, SBP, DBP, TG, LDL-C, prevalence of antilipidemic medication, FPG, SUA, ALT, and GGT were significantly high in the high BMI group, but HDL-C was low in the low BMI group. There were no inter-group differences regarding prevalence of alcohol consumption, smoking status, history of CVD, prevalence of antidiabetic medication, and eGFR.

Among them, the respective prevalence of normotension, prehypertension, and hypertension was $19.5 \%$ and $53.7 \%$, and $19.8 \%$. The prevalence of normotension and prehypertension decreased with increasing BMI and the prevalence of hypertension increased with increasing BMI (Fig. 1).

In addition to their direct associations, we observed a synergistic effect between BMI category and SUA levels on BP status in Fig. 2. In BMI $<21.0 \mathrm{~kg} / \mathrm{m}^{2}$, SUA correlated positively with both SBP and DBP $(r=0.112$, $p=0.100$ and $r=0.163, p=0.016$, respectively), but in BMI $\geq 25.0 \mathrm{~kg} / \mathrm{m}^{2}$, SUA correlated negatively with both $\mathrm{SBP}$ and DBP $(r=-0.178, p=0.023$ and $r=-0.064, p=$ 0.421 , respectively). Analysis of covariance showed that three regression lines in each graph were significantly different (SBP, $\mathrm{F}=8.139, p=0.004$ and DBP, $\mathrm{F}=5.199, p=$ 0.023 , respectively).

Table 2 shows the background characteristics of participants categorized by BMI (i.e., $<21.0 \mathrm{~kg} / \mathrm{m}^{2}$ and $\geq 21.0 \mathrm{~kg} / \mathrm{m}^{2}$ ) and blood pressure status. In hypertensive group with a BMI $<21.0 \mathrm{~kg} / \mathrm{m}^{2}$, prevalence of smoking status and GGT were significantly higher than normotensive group. In hypertensive group with a BMI $\geq 21.0 \mathrm{~kg} / \mathrm{m}^{2}$, age and BMI as well as smoking status and GGT were also significantly higher, but SUA was significantly lower than normotensive group.

Table 3 shows the relationship between various characteristics and blood pressure status of participants categorized by BMI. Age-adjusted partial Pearson's correlation coefficient showed that SUA correlated positively with both SBP and DBP in participants with a BMI $<21.0 \mathrm{~kg} / \mathrm{m}^{2}$ but correlated negatively with SBP in participants with a BMI $\geq 21.0 \mathrm{~kg} / \mathrm{m}^{2}$.

Table 4 shows multivariate-adjusted relationship between various characteristics and blood pressure status of participants categorized by BMI. Multiple linear regression analysis showed that SUA was significantly and positively associated with both SBP and DBP in participants with a $\mathrm{BMI}<21.0 \mathrm{~kg} / \mathrm{m}^{2}$, but negatively associated with both SBP and DBP in participants with a BMI $\geq 21.0 \mathrm{~kg} /$ $\mathrm{m}^{2}$, independently of other confounding factors.

We assessed the statistical significance of the synergistic relationship using a general linear model with the following confounding factors: age, BMI, alcohol consumption, smoking status, history of CVD, TG, HDL-C, LDL-C, prevalence of antilipidemic medication, FPG, prevalence of antidiabetic medication, SUA, eGFR, ALT, GGT, and the interaction between BMI and SUA (Table 5). The interaction between increased BMI and SUA as well as BMI and SUA were significant and independent determinants for $\operatorname{SBP}(\beta=-1.125, p=0.001)$ and DBP $(\beta=-0.995, P=$ $0.005)$, independently of other confounding factors.

Table 6 shows the odds ratios (ORs) $\{95 \%$ confidence interval (CI) $\}$ of SUA for blood pressure status of participants categorized by BMI. In participants with a BMI $\geq 21.0 \mathrm{~kg} / \mathrm{m}^{2}$, the odds ratio of SUA for hypertension was 0.75 (95\% CI, 0.59-0.95) compared with normotension, and $0.82(0.70-0.96)$ for hypertension compared with prehypertension. In subjects with a $\mathrm{BMI}<21.0 \mathrm{~kg} / \mathrm{m}^{2}$, these associations were not shown.

\section{Discussion}

In this cross-sectional, population-based study of 817 middle-aged and elderly men, we set out to determine 
Table 1 Characteristics of participants categorized by body mass index

\begin{tabular}{|c|c|c|c|c|}
\hline \multirow{3}{*}{ Characteristics N=817 } & \multicolumn{4}{|c|}{ Body mass index category $\left(\mathrm{kg} / \mathrm{m}^{2}\right)$} \\
\hline & \multirow{2}{*}{$\begin{array}{l}<21.0 \\
N=216\end{array}$} & \multirow{2}{*}{$\begin{array}{l}21.0-24.9 \\
N=439\end{array}$} & \multirow{2}{*}{$\begin{array}{l}\geq 25.0 \\
N=162\end{array}$} & \multirow[t]{2}{*}{$P$-value } \\
\hline & & & & \\
\hline Age (years) & $67 \pm 9$ & $67 \pm 9$ & $63 \pm 9$ & $<0.001$ \\
\hline Body mass index $\left(\mathrm{kg} / \mathrm{m}^{2}\right)$ & $19.5 \pm 1.1$ & $23.0 \pm 1.1$ & $27.0 \pm 2.0$ & $<0.001$ \\
\hline Alcohol consumption ${ }^{\mathrm{a}}(\%)$ & $37.0 / 20.8 / 9.3 / 32.9$ & $42.6 / 21.9 / 9.1 / 26.4$ & $34.6 / 30.2 / 7.4 / 27.8$ & 0.157 \\
\hline Smoking status ${ }^{\mathrm{b}}(\%)$ & 25.5/30.6/25.9/18.1 & $25.1 / 27.6 / 26.4 / 21.0$ & 23.5/28.4/24.7/23.5 & 0.910 \\
\hline History of CVD, N (\%) & $16(7.4)$ & $41(9.3)$ & $11(6.8)$ & 0.514 \\
\hline Systolic blood pressure $(\mathrm{mmHg})$ & $132 \pm 21$ & $136 \pm 19$ & $142 \pm 18$ & $<0.001$ \\
\hline Diastolic blood pressure (mmHg) & $79 \pm 11$ & $82 \pm 10$ & $85 \pm 11$ & $<0.001$ \\
\hline Triglycerides (mg/dL) & 79 (59-101) & $95(72-141)$ & $119(81-161)$ & $<0.001$ \\
\hline HDL cholesterol (mg/dL) & $67 \pm 17$ & $58 \pm 15$ & $53 \pm 13$ & $<0.001$ \\
\hline LDL cholesterol (mg/dL) & $103 \pm 28$ & $114 \pm 30$ & $115 \pm 33$ & $<0.001$ \\
\hline Antilipidemic medication, N (\%) & $3(1.4)$ & $25(5.7)$ & $9(5.6)$ & 0.035 \\
\hline Fasting plasma glucose (mg/dL) & $98(91-114)$ & $102(93-117)$ & $103(94-120)$ & 0.042 \\
\hline Antiidiabetic medication, N (\%) & $6(2.8)$ & $28(6.4)$ & $8(4.9)$ & 0.145 \\
\hline Serum uric acid (mg/dL) & $5.3 \pm 1.2$ & $5.7 \pm 1.3$ & $5.9 \pm 1.2$ & $<0.001$ \\
\hline eGFR $\left(\mathrm{ml} / \mathrm{min} . / 1.73 \mathrm{~m}^{2}\right)$ & $79.8 \pm 13.9$ & $77.0 \pm 14.3$ & $78.6 \pm 15.3$ & 0.051 \\
\hline Aspartate transaminase (IU/L) & $17(14-23)$ & $19(15-25)$ & $23(17-32)$ & $<0.001$ \\
\hline Y-glutamyl transpeptidase (IU/L) & $29(20-43)$ & $30(22-49)$ & $44(28-69)$ & $<0.001$ \\
\hline
\end{tabular}

Data for triglycerides, fasting plasma glucose, aspartate transaminase, and $\gamma$-glutamyl transpeptidase were skewed and are presented as median (interquartile range) values, and were log-transformed for analysis. $P$-value: Student's $\mathrm{t}$-test for continuous variables or the $\mathrm{x}^{2}$-test for categorical variables. Bolded numbers indicate significance

CVD cardiovascular disease, HDL high-density lipoprotein, LDL low-density lipoprotein, eGFR estimated glomerular filtration rate. Data presented are mean \pm standard deviation

${ }^{a}$ Daily alcohol consumption was measured using the Japanese liquor unit in which a unit corresponds to $22.9 \mathrm{~g}$ of ethanol, and the participants were classified into never, occasional, light daily ( $<2$ unit/day), and heavy daily drinkers ( $\geq 2$ unit/day)

${ }^{\mathrm{b} S}$ Smoking status [never-smoker, past-smoker, light smoker $(<30$ pack $\cdot$ year $)$, and heavy smoker $(\geq 30$ pack $\cdot$ year $\left.)\right]$
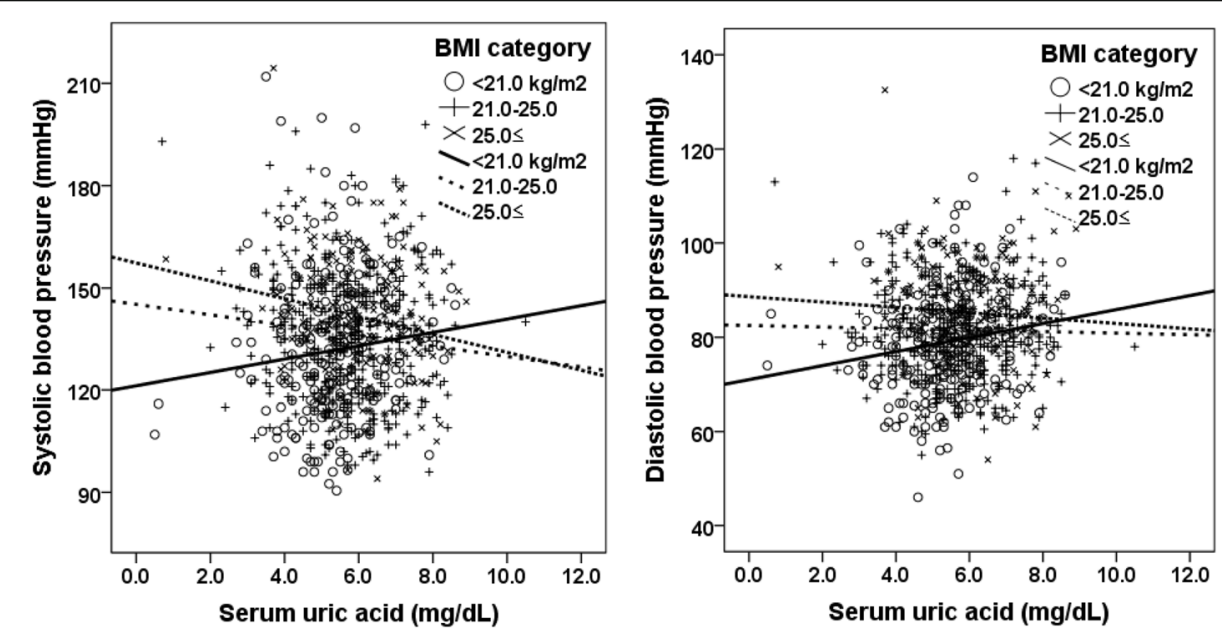

Fig. 2 Correlation between serum uric acid and blood pressure status of participants categorized by body mass index. In body mass index (BMI) $<21.0 \mathrm{~kg} / \mathrm{m}^{2}$, serum uric acid correlated positively with both systolic blood pressure (SBP) and diastolic blood pressure (DBP) $(r=0.112, p=0.100$ and $r=0.163, p=0.016$, respectively), but in BMl $\geq 21.0 \mathrm{~kg} / \mathrm{m}^{2}$ serum uric acid correlated negatively with both SBP and DBP (BMI $21-25 \mathrm{~kg} / \mathrm{m}^{2}, r=$ $-0.108, p=0.024$ and $r=-0.022, p=0.651 ; \mathrm{BMI} \geq 25.0 \mathrm{~kg} / \mathrm{m}^{2}, r=-0.178, p=0.023$ and $r=-0.064, p=0.421$, respectively). Analysis of covariance showed that three regression lines in each graph were significantly different (SBP, $F=8.139, P=0.004$ and $D B P, F=5.199, P=0.023$, respectively) 
Table 2 Characteristics of participants categorized by body mass index and blood pressure status

\begin{tabular}{|c|c|c|c|c|c|c|c|c|}
\hline \multirow{3}{*}{$\begin{array}{l}\text { Characteristics } \\
N=817\end{array}$} & \multicolumn{3}{|c|}{ Body mass index $<21.0 \mathrm{~kg} / \mathrm{m}^{2} \mathrm{~N}=216$} & \multirow{3}{*}{$\begin{array}{l}P \text { - } \\
\text { value }\end{array}$} & \multicolumn{3}{|c|}{ Body mass index $\geq 21.0 \mathrm{~kg} / \mathrm{m}^{2} \mathrm{~N}=601$} & \multirow{3}{*}{$P$-value } \\
\hline & Normotension & Prehypertension & Hypertension & & \multirow{2}{*}{$\begin{array}{l}\text { Normotension } \\
N=97\end{array}$} & \multirow{2}{*}{$\begin{array}{l}\text { Prehypertension } \\
N=245\end{array}$} & \multirow{2}{*}{$\begin{array}{l}\text { Hypertension } \\
N=259\end{array}$} & \\
\hline & $N=62$ & $N=90$ & $N=64$ & & & & & \\
\hline Age (years) & $65 \pm 10$ & $69 \pm 9$ & $68 \pm 8$ & 0.083 & $63 \pm 8$ & $67 \pm 9$ & $66 \pm 9$ & 0.002 \\
\hline Body mass index $\left(\mathrm{kg} / \mathrm{m}^{2}\right)$ & $19.4 \pm 1.0$ & $19.6 \pm 1.1$ & $19.6 \pm 1.1$ & 0.341 & $23.6 \pm 2.1$ & $23.7 \pm 2.1$ & $24.5 \pm 2.4$ & $<0.001$ \\
\hline Alcohol consumption (\%) & $\begin{array}{l}40.3 / 21.0 / 11.2 / \\
27.4\end{array}$ & $\begin{array}{l}34.4 / 27.8 / 10.0 / \\
27.8\end{array}$ & $\begin{array}{l}37.5 / 10.9 / 6.3 / \\
45.3\end{array}$ & 0.099 & $\begin{array}{l}43.3 / 19.6 / 10.3 / \\
26.8\end{array}$ & $\begin{array}{l}37.1 / 27.3 / 8.6 / \\
26.9\end{array}$ & $\begin{array}{l}42.5 / 22.8 / 8.1 / \\
26.6\end{array}$ & 0.731 \\
\hline Smoking status (\%) & $\begin{array}{l}30.6 / 37.1 / 21.0 / \\
11.3\end{array}$ & $\begin{array}{l}26.7 / 28.9 / 32.2 / \\
12.2\end{array}$ & $\begin{array}{l}18.8 / 26.6 / 21.9 / \\
32.8\end{array}$ & 0.011 & $\begin{array}{l}22.7 / 30.9 / 25.8 / \\
20.6\end{array}$ & $\begin{array}{l}30.6 / 30.2 / 22.4 / \\
16.7\end{array}$ & $\begin{array}{l}19.7 / 24.3 / 29.3 / \\
26.6\end{array}$ & 0.012 \\
\hline History of CVD, N (\%) & $3(4.8)$ & $10(11.1)$ & $3(4.7)$ & 0.214 & $6(6.2)$ & $28(11.4)$ & $18(6.9)$ & 0.130 \\
\hline Triglycerides (mg/dL) & $79(57-97)$ & $79(63-100)$ & $80(62-108)$ & 0.266 & $110(74-151)$ & $99(74-144)$ & $100(72-146)$ & 0.997 \\
\hline HDL cholesterol (mg/dL) & $68 \pm 17$ & $64 \pm 16$ & $70 \pm 18$ & 0.110 & $55 \pm 16$ & $57 \pm 15$ & $58 \pm 14$ & 0.278 \\
\hline LDL cholesterol (mg/dL) & $101 \pm 27$ & $102 \pm 28$ & $108 \pm 31$ & 0.327 & $118 \pm 29$ & $114 \pm 30$ & $114 \pm 33$ & 0.396 \\
\hline $\begin{array}{l}\text { Antilipidemic medication, } \\
\mathrm{N}(\%)\end{array}$ & 0 & $2(2.2)$ & $1(1.6)$ & 0.511 & $6(6.2)$ & $17(6.9)$ & $11(4.2)$ & 0.413 \\
\hline $\begin{array}{l}\text { Fasting plasma glucose } \\
(\mathrm{mg} / \mathrm{dL})\end{array}$ & $95(89-111)$ & $97(89-112)$ & $102(94-120)$ & 0.213 & $102(92-116)$ & $103(91-117)$ & $102(94-120)$ & 0.141 \\
\hline $\begin{array}{l}\text { Antiidiabetic medication, } \\
\text { N (\%) }\end{array}$ & $4(6.5)$ & 0 & $2(3.1)$ & 0.058 & $3(3.1)$ & $18(7.3)$ & $15(5.8)$ & 0.322 \\
\hline Serum uric acid (mg/dL) & $5.1 \pm 1.1$ & $5.4 \pm 1.3$ & $5.6 \pm 1.2$ & 0.059 & $6.0 \pm 1.1$ & $5.8 \pm 1.3$ & $5.6 \pm 1.3$ & 0.044 \\
\hline eGFR (ml/min./1.73m²) & $81.0 \pm 14.8$ & $78.3 \pm 12.9$ & $80.9 \pm 14.4$ & 0.372 & $76.8 \pm 13.4$ & $76.4 \pm 14.5$ & $78.5 \pm 15.1$ & 0.252 \\
\hline $\begin{array}{l}\text { Aspartate transaminase } \\
\text { (IU/L) }\end{array}$ & $19(15-24)$ & $17(13-22)$ & $18(14-24)$ & 0.102 & $18(15-24)$ & $20(15-26)$ & $20(16-27)$ & 0.084 \\
\hline $\begin{array}{l}\text { Y-glutamyl transpeptidase } \\
(\mathrm{IU} / \mathrm{L})\end{array}$ & $27(20-37)$ & $27(19-38)$ & $35(21-64)$ & 0.004 & $29(21-43)$ & $34(22-50)$ & $36(25-65)$ & $<0.001$ \\
\hline
\end{tabular}

Data for triglycerides, fasting plasma glucose, aspartate transaminase, and $\gamma$-glutamyl transpeptidase were skewed and were log-transformed for analysis. $P$-value: Student's t-test for continuous variables or the $\mathrm{X}^{2}$-test for categorical variables. Bolded numbers indicate significance

Table 3 Age-adjusted relationship between various characteristics and blood pressure status of participants categorized by body mass index

\begin{tabular}{|c|c|c|c|c|}
\hline \multirow[b]{3}{*}{ Characteristics $N=817$} & \multicolumn{2}{|c|}{ Body mass index $<21.0 \mathrm{~kg} / \mathrm{m}^{2} \mathrm{~N}=216$} & \multicolumn{2}{|c|}{ Body mass index $\geq 21.0 \mathrm{~kg} / \mathrm{m}^{2} \mathrm{~N}=601$} \\
\hline & Systolic blood pressure & Diastolic blood pressure & Systolic blood pressure & Diastolic blood presure \\
\hline & Partial r ( $P$-value) & Partial r (P-value) & Partial r ( $P$-value) & Partial r (P-value) \\
\hline Body mass index & $0.105(0.127)$ & $0.093(0.174)$ & $0.190(<0.001)$ & $0.153(<0.001)$ \\
\hline Alcohol consumption & $0.137(0.044)$ & $0.107(0.119)$ & $-0.008(0.844)$ & $-0.030(0.469)$ \\
\hline Smoking status & $0.205(0.003)$ & $0.245(<0.001)$ & $0.142(<0.001)$ & $0.156(<0.001)$ \\
\hline History of CVD (Yes = 1, No=0) & $-0.061(0.371)$ & $-0.020(0.766)$ & $-0.051(0.216)$ & $-0.051(0.209)$ \\
\hline Triglycerides & $0.047(0.489)$ & $0.112(0.100)$ & $0.031(0.446)$ & $0.064(0.116)$ \\
\hline HDL cholesterol & $0.078(0.255)$ & $0.081(0.240)$ & $0.054(0.186)$ & $0.061(0.138)$ \\
\hline LDL cholesterol & $-0.027(0.689)$ & $0.057(0.404)$ & $-0.019(0.643)$ & $0.000(0.994)$ \\
\hline Antilipidemic medication ( $\mathrm{Yes}=1, \mathrm{No}=0$ ) & $0.068(0.318)$ & $0.018(0.791)$ & $-0.098(0.017)$ & $-0.040(0.334)$ \\
\hline Fasting plasma glucose & $0.138(0.043)$ & $0.059(0.392)$ & $0.113(0.005)$ & $0.029(0.476)$ \\
\hline Antiidiabetic medication ( $\mathrm{Yes}=1, \mathrm{No}=0$ ) & $-0.056(0.410)$ & $-0.064(0.349)$ & $0.007(0.871)$ & $-0.051(0.214)$ \\
\hline Serum uric acid & $0.134(0.049)$ & $0.153(0.025)$ & $-0.095(0.020)$ & $-0.034(0.401)$ \\
\hline Estimated GFR & $0.184(0.007)$ & $0.083(0.223)$ & $0.083(0.041)$ & $0.015(0.719)$ \\
\hline Aspartate transaminase & $0.001(0.983)$ & $0.007(0.917)$ & $0.084(0.040)$ & $0.080(0.051)$ \\
\hline Y-glutamyl transpeptidase & $0.184(0.007)$ & $0.240(<0.001)$ & $0.171(<0.001)$ & $0.202(<0.001)$ \\
\hline
\end{tabular}

r, Pearson's partial correlation coefficients adjusted for age. Data for triglycerides, fasting plasma glucose, aspartate transaminase, and $\gamma$-glutamyl transpeptidase were skewed and were log-transformed for analysis. Bolded numbers indicate significance 
Table 4 Multivariate-adjusted relationship between various characteristics and blood pressure status of participants categorized by body mass index

\begin{tabular}{|c|c|c|c|c|}
\hline \multirow[b]{3}{*}{ Characteristics $N=817$} & \multicolumn{2}{|c|}{ Body mass index category $<21.0 \mathrm{~kg} / \mathrm{m}^{2} N=216$} & \multicolumn{2}{|c|}{ Body mass index category $\geq 21.0 \mathrm{~kg} / \mathrm{m}^{2} \mathrm{~N}=601$} \\
\hline & Systolic blood pressure & Diastolic blood pressure & Systolic blood pressure & Diastolic blood pressure \\
\hline & $\beta(P-v a l u e) \S$ & $\beta(P-v a l u e) \S$ & $\beta(P-v a l u e) \S$ & $\beta(P-v a l u e) \S$ \\
\hline Age & $0.375(<0.001)$ & $0.065(0.396)$ & $0.275(<0.001)$ & $-0.045(0.332)$ \\
\hline Body mass index & - & - & $0.175(<0.001)$ & $0.141(0.001)$ \\
\hline Alcohol consumption & $0.124(0.064)$ & - & - & $-0.046(0.247)$ \\
\hline Smoking status & $0.175(0.010)$ & $0.182(0.014)$ & $0.114(0.006)$ & $0.102(0.020)$ \\
\hline History of CVD (Yes = 1, No=0) & - & - & - & $-0.042(0.307)$ \\
\hline Triglycerides & - & - & - & $0.064(0.167)$ \\
\hline HDL cholesterol & - & - & - & $0.083(0.067)$ \\
\hline LDL cholesterol & - & $0.162(0.018)$ & - & - \\
\hline Antilipidemic medication (Yes $=1, \mathrm{No}=0$ ) & - & - & $-0.090(0.020)$ & - \\
\hline Fasting plasma glucose & $0.151(0.020)$ & $0.087(0.197)$ & $0.088(0.024)$ & - \\
\hline Antiidiabetic medication (Yes $=1, \mathrm{No}=0$ ) & - & $-0.060(0.370)$ & - & $-0.041(0.302)$ \\
\hline Serum uric acid & $0.178(0.011)$ & $0.155(0.028)$ & $-0.127(0.001)$ & $-0.102(0.019)$ \\
\hline Estimated GFR & $0.272(<0.001)$ & $0.170(0.030)$ & - & $-0.036(0.429)$ \\
\hline Aspartate transaminase & - & $-0.086(0.243)$ & - & - \\
\hline ү-glutamyl transpeptidase & - & $0.243(0.002)$ & $0.124(0.007)$ & $0.157(0.001)$ \\
\hline$R^{2}$ & $0.167(<0.001)$ & $0.163(<0.001)$ & $0.127(<0.001)$ & $0.084(<0.001)$ \\
\hline
\end{tabular}

Only factors remained in the final model were shown. Data for triglycerides, fasting plasma glucose, aspartate transaminase, and $\gamma$-glutamyl transpeptidase were skewed and were log-transformed for analysis. Bolded numbers indicate significance

$\beta$ standard coefficient, $R^{2}$ coefficient of determination

§Multivariate adjusted for all confounding factors in Table 1 by multiple linear regression analysis using backward elimination method

the prevalence of prehypertension and hypertension, as defined by the JNC-7 criteria [1], and its association with BMI and SUA. This study showed that increased SUA levels were positively associated with SBP and DBP in participants with a BMI $<21.0 \mathrm{~kg} /$ $\mathrm{m}^{2}$, but negatively in those with a BMI $\geq 21.0 \mathrm{~kg} / \mathrm{m}^{2}$.
The effect of significant interaction between BMI and SUA on blood pressure indicated that increased SUA was a risk factor for elevated blood pressure in underweight participants, but was not a predictor among normal- or overweight participants. To our knowledge, few studies have indicated that BMI may

Table 5 Interaction between body mass index and uric acid on blood pressure status

\begin{tabular}{|c|c|c|}
\hline & Systolic blood pressure & Diastolic blood pressure \\
\hline Characteristics $N=817$ & $\beta(P$-value $) \S$ & $\beta(P$-value $) \S$ \\
\hline Age & $0.294(<0.001)$ & - \\
\hline Body mass index & $0.715(<0.001)$ & $0.620(<0.001)$ \\
\hline Smoking status & $0.117((0.001)$ & $0.137(<0.001)$ \\
\hline LDL cholesterol & - & $0.057(0.096)$ \\
\hline Fasting plasma glucose & $0.092(0.006)$ & - \\
\hline Serum uric acid & $0.881(0.002)$ & $0.776(0.008)$ \\
\hline Estimated GFR & $0.092(0.016)$ & - \\
\hline Y-glutamyl transpeptidase & $0.119(0.002)$ & $0.178(<0.001)$ \\
\hline Body mass index ${ }^{a}$ serum uric acid & $-1.125(0.001)$ & $-0.995(0.005)$ \\
\hline$R^{2}$ & $0.148(<0.001)$ & $0.118(0<0.001)$ \\
\hline
\end{tabular}

Data for fasting plasma glucose, triglycerides, aspartate transaminase, and $\gamma$-glutamyl transpeptidase were skewed and were log-transformed for analysis. Bolded numbers indicate significance

$\beta$ standard coefficient, $R^{2}$ coefficient of determination

§Multivariate adjusted for all confounding factors in Table 1 by multiple linear regression analysis using backward elimination method. Only factors remained in the final model were shown

anteraction between body mass index and serum uric acid 
Table 6 Association between serum uric acid levels and blood pressure status of participants categorized by body mass index

\begin{tabular}{|c|c|c|c|c|c|c|c|}
\hline \multirow{2}{*}{$\begin{array}{l}\text { Characteristic } \\
N=817\end{array}$} & \multicolumn{3}{|c|}{ Body mass index $<21.0 \mathrm{~kg} / \mathrm{m}^{2}$} & \multicolumn{3}{|c|}{ Body mass index $\geq 21.0 \mathrm{~kg} / \mathrm{m}^{2}$} & \multirow{2}{*}{$\begin{array}{l}P \text { - } \\
\text { interaction }\end{array}$} \\
\hline & $N=216$ & OR $(95 \% \mathrm{Cl})$ & $P$-value $\S$ & $N=601$ & OR $(95 \% \mathrm{Cl})$ & $P$-value $\S$ & \\
\hline \multicolumn{8}{|c|}{ Prehypertension VS Normotension } \\
\hline Serum uric acid & $90 / 62$ & $1.25(0.91-1.72)$ & 0.168 & $245 / 97$ & $0.95(0.77-1.18)$ & 0.650 & 0.073 \\
\hline \multicolumn{8}{|c|}{ Hypertension VS Normotension } \\
\hline Serum uric acid & $64 / 62$ & $1.53(0.99-2.38)$ & 0.058 & $259 / 97$ & $0.75(0.59-0.95)$ & 0.018 & 0.004 \\
\hline \multicolumn{8}{|c|}{ Hypertension VS Prehypertension } \\
\hline Serum uric acid & $64 / 90$ & $1.26(0.92-1.74)$ & 0.149 & $259 / 245$ & $0.82(0.70-0.96)$ & 0.012 & 0.077 \\
\hline
\end{tabular}

modify the association between SUA and blood pressure status.

In men aged $<40$ years, SUA was significantly associated with $\operatorname{SBP}(\beta=0.25, p=0.002)$ and $\operatorname{DBP}(\beta=0.41, p<$ 0.001 ) after adjustment for age, diabetes, dyslipidemia, BMI, and eGFR, but the association was not significant in those $\geq 60$ years [21]. From a meta-analysis of 25 studies with 97,824 participants assessing the association between UA and incident hypertension, it was suggested that hyperuricemia may modestly increase the risk of hypertension incidence [22]. From a meta-analysis of 18 prospective cohort studies representing data from 55,607 participants, it was shown that hyperuricemia is associated with an increased risk for incident hypertension, independent of traditional hypertension risk factors. This risk appears to be more pronounced in younger individuals and women [12]. Thus, one can suggest that hyperuricemia-related pathogenetic mechanisms may be more dominant in earlier stages of hypertension than later stages when salt-sensitivity becomes apparent [12]. Our study findings indicate that we should consider the effect of BMI as well as age on the relationship between SUA and blood pressure.

The mechanism whereby BMI may modify the association between SUA and blood pressure status are not completely understood. SUA induces endothelial cell dysfunction via nitric oxide (NO) synthetase [23] and directly involves stimulation of the renin-angiotensin system [24]. SUA alters the proliferation/migration of endothelial and vascular smooth muscle cells [25]. These findings may provide insight into a pathogenic mechanism by which UA may induce hypertension and vascular disease [26]. A recent rodent model with induced hyperuricemia demonstrated that UA might have a pathogenic role in the development of renal afferent arteriolopathy and tubulointerstitial disease, leading to hypertension [27]. The renal lesions and the development of hypertension were prevented by lowering UA levels with allopurinol or benziodarone, which inhibits xanthine oxidase and hence blocks both UA and oxidant formation, which are reversed by angiotensin-converting enzyme inhibition [28].

Moreover, SUA also reflects systemic inflammation [29], oxidative stress [30] and is more strongly associated with insulin resistance [31,32] and other cardiovascular risk factors such as BMI, BP, T-C, HDL-C, TG, and FPG $[31,33]$. Increased BMI is also significantly associated with various CVD risk factors. These risk factors cause endothelial dysfunction [34], the loss of vasomotor reactivity [35] and arterial stiffness [36]. Such pathophysiology induced by increased BMI may be greater than that of SUA. Thus, SUA could not be an independent risk factor for increased SBP in participants with a BMI $\geq 21.0 \mathrm{~kg} / \mathrm{m}^{2}$. We suggest that SUA may be more important in participants with a BMI $<21.0 \mathrm{~kg}$. It has been demonstrated that increased SUA induces increased BP that is initially reversible but leads to an irreversible salt-sensitive hypertension over time [37]. Thus, SUA identification is important for risk assessment and treatment of such patients with a BMI $<21.0 \mathrm{~kg}$.

An important observation was that UA may function as an antioxidant, and possibly one of the most important antioxidants in plasma. Increased SUA in subjects with CVD might therefore reflect a compensatory mechanism to counter the oxidative stress that occurs in these conditions [38]. In our study, SUA was negatively associated with hypertension in participants with a BMI $\geq 21.0 \mathrm{~kg} / \mathrm{m}^{2}$. The beneficial antioxidant actions of SUA may partially counter its potential detrimental effects. It is interesting that almost all studies examining the relation of SUA levels with CVD events show a J-shaped curve with the nadir of risk in the second quartile $[39,40]$.

Some limitations of this study must be considered. First, our cross-sectional study design does not eliminate potential causal relationships between BMI, SUA, and blood pressure status. Second, the prevalence of blood pressure categories is based on single blood pressure measurement. Third, confounding factors are based on a single assessment of blood, which may introduce a misclassification bias. Fourth, we could not eliminate the 
possible effects of underlying diseases and medications for diabetes and dyslipidemia on the present findings. We could not rule out one-time prehypertension and white-coat prehypertension. Finally, in this study, the demographics and referral source may limit the generalization of the results.

\section{Conclusion}

The present study showed that BMI may modify the association between SUA, SBP, and DBP. The underlying mechanism seems to be independent from traditional cardiovascular risk factors such as age, BMI, alcohol consumption, smoking status, history of CVD, lipids, diabetes, renal function, and liver function. For community-dwelling healthy persons, prospective population-based studies are needed to investigate the mechanisms underlying this association to determine whether intervention, such as effective lifestyle modifications that decrease BMI and SUA, in adult populations will decrease risks.

\section{Acknowledgements}

This work was supported in part by a grant-in-aid for Scientific Research from the Foundation for Development of Community (2017)

\section{Funding}

This work was supported in part by a grant-in-aid from the Foundation for Development of Community (2017).

\section{Availability of data and materials}

Not applicable.

\section{Authors' contributions}

RK participated in the design of the study, performed the statistical analysis and drafted the manuscript. RK DN, SK, and TeK contributed to the acquisition and interpretation of data. RK, DN, and TeK contributed to the conception and design of the statistical analysis. All authors read and approved the manuscript.

\section{Ethics approval and consent to participate}

This study was approved by the ethics committee of Ehime University School of Medicine, and written informed consent was obtained from each subject.

\section{Consent for publication}

The author has given all rights for this manuscript to the publisher.

\section{Competing interests}

The authors declare that they have no competing interests.

\section{Publisher's Note}

Springer Nature remains neutral with regard to jurisdictional claims in published maps and institutional affiliations.

Received: 25 October 2017 Accepted: 23 January 2018

Published online: 30 January 2018

\section{References}

1. Chobanian AV, Bakris GL, Black HR, Cushman WC, Green LA, Izzo JL Jr, et al. Joint National Committee on prevention, detection, evaluation, and treatment of high blood pressure. National Heart, Lung, and Blood Institute; National High Blood Pressure Education Program Coordinating Committee: the seventh report of the joint National Committee on prevention, detection, evaluation, and treatment of high blood pressure. Hypertension. 2003:42(6):1206-52

2. Gu D, Chen J, Wu X, Duan X, Jones DW, Huang JF, et al. Prehypertension and risk of cardiovascular disease in Chinese adults. J Hypertens. 2009;27(4): 721-9.

3. Kawamoto R, Kohara K, Tabara Y, Miki T. High prevalence of prehypertension is associated with the increased body mass index in community-dwelling Japanese. Tohoku J Exp Med. 2008;216(4):353-61.

4. Isezuo SA, Sabir AA, Ohwovorilole AE, Fasanmade OA. Prevalence, associated factors and relationship between prehypertension and hypertension: a study of two ethnic African populations in northern Nigeria. J Hum Hypertens. 2011;25(4):224-30.

5. Zhang W, Li N. Prevalence, risk factors, and management of prehypertension. Int J Hypertens. 2011;2011:605359.

6. Kawamoto R, Tabara Y, Kohara K, Miki T, Abe M, Kusunoki T. Increased highdensity lipoprotein cholesterol is associated with a high prevalence of prehypertension and hypertension in community-dwelling persons. Endocrine. 2012;42(2):321-8.

7. Ishikawa Y, Ishikawa J, Ishikawa S, Kayaba K, Nakamura Y, Shimada K, et al. Jichi medical school cohort investigators group. Hypertens Res. 2008;31(7): 1323-30.

8. Lewington S, Clarke R, Qizilbash N, Peto R, Collins R, Prospective Studies Collaboration. Age-specific relevance of usual blood pressure to vascular mortality: a meta-analysis of individual data for one million adults in 61 prospective studies. Lancet. 2002;360(9349):1903-13.

9. Jin M, Yang F, Yang I, Yin Y, Luo JJ, Wang H, et al. Uric acid, hyperuricemia and vascular diseases. Front Biosci (Landmark Ed). 2012;17:656-69.

10. Bao X, Wang Q, Chen G, Wang Q, Liang R, Zhang S. Serum concentration of uric acid associated with prehypertension among Chinese population. Angiology. 2014;65(9):800-5.

11. Jiang M, Gong D, Fan Y. Serum uric acid levels and risk of prehypertension: a meta-analysis. Clin Chem Lab Med. 2017;55(3):314-21.

12. Grayson PC, Kim SY, LaValley M, Choi HK. Hyperuricemia and incident hypertension: a systematic review and meta-analysis. Arthritis Care Res (Hoboken). 2011;63(1):102-10.

13. Yuan H, Yu C, Li X, Sun L, Zhu X, Zhao C, et al. Serum uric acid levels and risk of metabolic syndrome: a dose-response meta-analysis of prospective studies. J Clin Endocrinol Metab. 2015;100(11):4198-207.

14. Zhao G, Huang L, Song M, Song Y. Baseline serum uric acid level as a predictor of cardiovascular disease related mortality and all-cause mortality: a meta-analysis of prospective studies. Atherosclerosis. 2013;231(1):61-8.

15. Puddu PE, Bilancio G, Terradura Vagnarelli O, Lombardi C, Mancini M, Zanchetti A, et al. Serum uric acid and eGFR_CKDEPI differently predict long-term cardiovascular events and all causes of deaths in a residential cohort. Int J Cardiol. 2014;171(3):361-7.

16. Lu Z, Dong B, Wu H, Chen T, Zhang Y, Wu J, et al. Serum uric acid level in primary hypertension among Chinese nonagenarians/centenarians. J Hum Hypertens. 2009;23(2):113-21.

17. Glantzounis GK, Tsimoyiannis EC, Kappas AM, Galaris DA. Uric acid and oxidative stress. Curr Pharm Des. 2005;11(32):4145-51.

18. Ishizaka N, Ishizaka Y, Toda A, Tani M, Koike K, Yamakado M, et al. Changes in waist circumference and body mass index in relation to changes in serum uric acid in Japanese individuals. J Rheumatol. 2010:37(2):410-6.

19. Kawamoto R, Ninomiya D, Kasai Y, Kusunoki T, Ohtsuka N, Kumagi T, et al. Handgrip strength is associated with metabolic syndrome among middleaged and elderly community-dwelling persons. Clin Exp Hypertens. 2016; 38(2):245-51.

20. Horio M, Imai E, Yasuda Y, Watanabe T, Matsuo S. Modification of the CKD epidemiology collaboration (CKD-EPI) equation for Japanese: accuracy and use for population estimates. Am J Kidney Dis. 2010;56(1):32-8.

21. Lee JJ, Ahn J, Hwang J, Han SW, Lee KN, Kim JB, et al. Relationship between uric acid and blood pressure in different age groups. Clin Hypertens. 2015; $21: 14$

22. Wang J, Qin T, Chen J, Li Y, Wang L, Huang H, et al. Hyperuricemia and risk of incident hypertension: a systematic review and meta-analysis of observational studies. PLoS One 2014:9(12):e114259.

23. Maxwell AJ, Bruinsma KA. Uric acid is closely linked to vascular nitric oxide activity. Evidence for mechanism of association with cardiovascular disease. J Am Coll Cardiol. 2001;38(7):1850-8.

24. Yu MA, Sánchez-Lozada LG, Johnson RJ, Kang DH. Oxidative stress with an activation of the renin-angiotensin system in human vascular endothelial 
cells as a novel mechanism of uric acid-induced endothelial dysfunction. $J$ Hypertens. 2010;28(6):1234-42.

25. Kang DH, Park SK, Lee IK, Johnson RJ. Uric acid-induced C-reactive protein expression: implication on cell proliferation and nitric oxide production of human vascular cells. J Am Soc Nephrol. 2005;16(12):3553-62.

26. Khosla UM, Zharikov S, Finch JL, Nakagawa T, Roncal C, Mu W, et al. Hyperuricemia induces endothelial dysfunction. Kidney Int. 2005;67(5): 1739-42.

27. Johnson RJ, Kang DH, Feig D, Kivlighn S, Kanellis J, Watanabe S, et al. Is there a pathogenetic role for uric acid in hypertension and cardiovascular and renal disease? Hypertension. 2003:41 (6):1183-90.

28. Mazzali M, Hughes J, Kim YG, Jefferson JA, Kang DH, Gordon KL, et al. Elevated uric acid increases blood pressure in the rat by a novel crystalindependent mechanism. Hypertension. 2001;38(5):1101-6.

29. Lyngdoh T, Marques-Vidal P, Paccaud F, Preisig M, Waeber G, Bochud M, et al. Elevated serum uric acid is associated with high circulating inflammatory cytokines in the population-based Colaus study. PLoS One. 2011:6(5):e19901.

30. Park JH, Jin YM, Hwang S, Cho DH, Kang DH, Jo I. Uric acid attenuates nitric oxide production by decreasing the interaction between endothelial nitric oxide synthase and calmodulin in human umbilical vein endothelial cells: a mechanism for uric acid-induced cardiovascular disease development. Nitric Oxide. 2013:32:36-42.

31. Vuorinen-Markkola H, Yki-Järvinen H. Hyperuricemia and insulin resistance. J Clin Endocrinol Metab. 1994;78(1):25-9.

32. Kawamoto R, Tabara Y, Kohara K, Miki T, Kusunoki T, Takayama S, et al. Usefulness of combining serum uric acid and high-sensitivity C-reactive protein for risk stratification of patients with metabolic syndrome in community-dwelling women. Endocrine. 2013;44(1):132-9.

33. Cigolini M, Targher G, Tonoli M, Manara F, Muggeo M, De Sandre G. Hyperuricaemia: relationships to body fat distribution and other components of the insulin resistance syndrome in 38-year-old healthy men and women. Int J Obes Relat Metab Disord. 1995;19(2):92-6.

34. Jagla A, Schrezenmeir J. Postprandial triglycerides and endothelial function. Exp Clin Endocrinol Diabetes. 2001;109(4):S533-47.

35. Lundman P, Eriksson M, Schenck-Gustafsson K, Karpe F, Tornvall P. Transient triglyceridemia decreases vascular reactivity in young, healthy men without risk factors for coronary heart disease. Circulation. 1997;96(10):3266-8.

36. Yao WM, Zhang HF, Zhu ZY, Zhou YL, Liang NX, Xu DJ, et al. Genetically elevated levels of circulating triglycerides and brachial-ankle pulse wave velocity in a Chinese population. J Hum Hypertens. 2013;27(4):265-70.

37. Watanabe S, Kang DH, Feng L, Nakagawa T, Kanellis J, Lan H, et al. Uric acid, hominoid evolution, and the pathogenesis of salt-sensitivity. Hypertension. 2002:40(3):355-60.

38. Nieto FJ, Iribarren C, Gross MD, Comstock GW, Cutler RG. Uric acid and serum antioxidant capacity: a reaction to atherosclerosis? Atherosclerosis. 2000;148(1):131-9

39. Iribarren C, Sharp DS, Curb JD, Yano K. High uric acid: a metabolic marker of coronary heart disease among alcohol abstainers? J Clin Epidemiol. 1996; 49(6):673-8

40. Fang J, Alderman MH. Serum uric acid and cardiovascular mortality: the NHANES I epidemiologic follow-up study, 1971-1992. JAMA. 2000;283(18): 2404-10.

\section{Submit your next manuscript to BioMed Central and we will help you at every step:}

- We accept pre-submission inquiries

- Our selector tool helps you to find the most relevant journal

- We provide round the clock customer support

- Convenient online submission

- Thorough peer review

- Inclusion in PubMed and all major indexing services

- Maximum visibility for your research

Submit your manuscript at www.biomedcentral.com/submit

) Biomed Central 